2016 - Volume: 17 Number: 2

Page: 391 - 400

DOI : $10.18038 /$ btda. 96384

Received: 07 April 2016

Revised: 06 June 2016 Accepted: 13 June 2016

\title{
SYNTHESIS OF CHITOSAN-BASED HYDROGEL BY USING PHOTOPOLYMERIZATION TECHNIQUE
}

\author{
Neslihan ALEMDAR \\ Department of Chemistry, Marmara University, 34722, İstanbul, TURKEY
}

\begin{abstract}
In this study, a novel route was developed to fabricate chitosan-based hydrogel by using photopolymerization technique. Firstly, glycidyl methacrylate (GMA) grafted onto the chitosan (CTS) backbone to produce chitosan derivative product having photopolymerizable groups (CTS-g-GMA). Following, CTS-g-GMA and PEGDA are subjected to photopolymerization to form chemically crosslinked hydrogel. The chemical structure of the obtained chitosan-based hydrogel was characterized by Fourier transform infrared (FT-IR) analyses. Thermal behavior of hydrogel was determined by thermal gravimetric analysis (TGA) and differential thermal analysis (DTA). The thermal analyses showed that (CTS-g-GMA)-PEGDA had higher thermal stability than neat chitosan. Water uptake capacities (swelling ratio) of hydrogels obtained by using different UV exposure time were determined gravimetrically. From the swelling test result, it was observed that exposure time has the effect on the water uptake capacity of hydrogels. Additionally, cytotoxicity test for chitosan-based hydrogel was carried out by using L929 fibroblasts. All of the obtained results demonstrated that chitosan-based hydrogel fabricated in this work could be used for biomedical applications in future works.
\end{abstract}

Keywords: Chitosan, Photopolymerization, Polymeric network, Hydrogel

\section{INTRODUCTION}

Hydrogels with three-dimensional (3D) materials have the highly water uptake capacity owing to its hydrophilic and porous structure while maintaining their dimensional stability. Hydrogels formed by either physical or chemical crosslinking process have been sparked confidential attention for biomedical applications since they have unique physical properties. Additionally, their porous structure can be tuned by controlling the crosslinking density in the gel matrix and correspondingly ability to absorb water (swelling ratio) in the aqueous environment [1-3]. Nowadays, hydrogels with superior features have been produced using biopolymers. Chitosan, a partially N-deacetylated product of chitin, is an important natural biopolymer and it is known as a hydrophilic and cationic polysaccharide. Since chitosan has excellent properties such as biodegradability, biocompatibility, non- toxicity and $\mathrm{pH}$-sensitive structure, it has been used with broad applications in biomedical, tissue engineering and drug delivery field [410]. Although chitosan has the mentioned advantages, there are some limitations in applications because of its rigid crystalline structure and the deacetylation resulting in its insoluble in alkali solutions. To overcome this drawback, various modification techniques have been successfully performed owing to amino groups of chitosan structure. The most important and effective modification method is grafting among these techniques [11]. Photopolymerizable groups are created on the chitosan backbone by means of grafting modification method. Owing to these groups, photopolymerization can be performed to form chitosan-based hydrogel having highly swollen three-dimensional structure [12]. Photopolymerization technique, which is used to convert a sol-state to a hydrogel form by free radical polymerization, has several advantages over conventional polymerization techniques because it provides i) polymerization in a fast and controllable manner under ambient or physiological conditions ii) spatial and temporal control over polymerization, iii) fast curing rates (from seconds to minutes) at room temperatures $[13,14]$. Moreover, degradation of sensitive molecules which decompose at a high reaction temperature is avoided since photopolymerization can be conducted at room temperature. Additionally,

Corresponding Author: neslihan.alemdar@marmara.edu.tr 
crosslinking density of hydrogels can be controlled by adjusting UV exposure time, UV exposure power, and photoinitiator concentration $[12,13,15]$. To utilize the advantages of photopolymerization technique, photopolymerizable chitosan which facilitates to compose chemically polymeric network has been synthesized by using different materials and used for a number of biomedical applications. For instance, di-acrylated pluronic and glycidyl methacrylated chitooligosaccharide were photo-irradiated to form hydrogel for wound healing applications [12]. In another work, photopolymerizable chitosan derivative was synthesized by chitosan and methyl acroloyl glycine (MAG) creating hydrogels with porous structure for tissue engineering [16]. A water-soluble chitosan derivative is favorable for biomedical applications. Glycol chitosan (GC), one of these kinds of derivative, has been produced to promote the solubility of chitosan in physiological solvents, and converted to a photopolymerizable polymer through methacrylation [17]. Photopolymerizable chitosan was also synthesized through styrenation and polymerized in the presence of camphorquinone (CQ) photoinitiator upon visible light irradiation [18]. As seen from all investigations, the enormous advantage of forming photopolymerizable chitosan refers to the opportunity to combine natural photopolymerizable polymers with synthetic biocompatible or degradable polymers to create new hybrid materials.

In current study, glycidyl methacrylate (GMA) was grafted onto the CTS backbone to functionalize CTS and to constitute polymerizable groups on CTS structure. GMA was chosen as a grafting agent because it has two reactive functional groups such as an epoxy group and an acrylic group. Additionally, GMA favorably reacts with CTS by minimizing steric hindrance that affects hydroxyl groups in the CTS backbone [19]. After this modification step of chitosan by grafting with GMA, CTS acquires photopolymerizable acryclic groups onto its backbone. GMA grafted chitosan (CTS-g-GMA) and poly(ethylene glycol) diacrylate (PEGDA) was subjected to photopolymerization to form crosslinked hydrogel. PEGDA with excellent properties such as biocompatible and highly low toxicity was used to fabricate hydrogel since it also behaves as a cross-linker during the formation of hydrogel. Moreover, PEGDA has not only hydrophilic structure but also elastic properties [20,21]. Structure of the obtained hydrogel was characterized by FT-IR analyses. Thermal behavior of the hydrogel was determined by thermal analyses (TGA and DTA). Swelling test of hydrogels was carried out in distilled water at $37^{\circ} \mathrm{C}$. From all of the obtained results, it can be visualized that the obtained hydrogel has a highly hydrophilic structure and accordingly a potential use as a biomedical material.

\section{MATERIAL AND METHODS}

\subsection{Materials}

Chitosan (CTS, $\mathrm{M}_{\mathrm{w}}=3 \times 10^{5} \mathrm{~g} / \mathrm{mol}$, degree of deacetylation: 87\%) was supplied by CID DESC (Mexico). Glycidyl methacrylate monomer (GMA), poly(ethylene glycol) diacrylate (PEGDA), 2-hydroxy-1-[4(hydroxyethoxy) phenyl]-2-methyl-1-propanone (Irgacure 2959), acetonitrile $\left(\mathrm{C}_{2} \mathrm{H}_{3} \mathrm{~N}\right)$, tetrahydrofuron (THF) were purchased from Aldrich (Milwaukee, WI, USA) and were used as received. Cell Culture Chemicals and solutions including medium (Dulbecco's Modified Eagle Medium-Roswell Park Memorial Institute, DMEM- RPMI), antibiotic, trypsin, fetal bovine serum (FBS) were purchased from PAN Biotech (Germany). (4-[3-(4-iodophenyl)-2-(4-nitrophenyl)-2H-5-tetrazolio] 1,3benzenedisulfonate) (WST-1) cell proliferation reagent was provided from Roche (Switzerland). L929 (Murine Fibroblasts) cell lines were received as gifts from Prof. Julide Akbuga (Marmara University).

\subsection{Synthesis of the CTS-g-GMA polymer}

CTS-g-GMA was synthesized as given in the literature [19]. Firstly, chitosan (1.0 g) was dissolved in acetic acid of $0.4 \mathrm{M}$ and then GMA was slowly and drop-by-drop added into this CTS solution. CTS/GMA molar ratio was taken as a 2:1. The $\mathrm{pH}$ of the reaction medium was adjusted to 3.8 by using $0.05 \mathrm{M} \mathrm{KOH}$. The grafting reaction was carried out at $60^{\circ} \mathrm{C}$ for 2 hours under nitrogen atmosphere. End of the reaction time, the solution was cooled in an ice bath for $15 \mathrm{~min}$ to stop the mixture from further reacting. The cooled solution was precipitated into the acetonitrile to obtain the final product, which is 
white solid with a thin fiber-like appearance. To remove any free poly(GMA) that could form from selfpolymerization, the obtained product with a white color was washed several times with a tetrahydrofuran (THF). After this purification step, the product was dried for $72 \mathrm{~h}$ at room temperature under the vacuum.

\subsection{Fabrication of Chitosan-Based Hydrogel by Photopolymerization}

To prepare $5 \%$ polymer solutions, CTS-g-GMA $(250 \mathrm{mg})$ obtained in the first step was completely dissolved in the $10 \mathrm{~mL}$ of distilled water. Following, PEGDA $(250 \mathrm{mg})$ and Irgacure $2959(0.5 \%$, w/v) were added as a crosslinker and photoinitatior, respectively. The prepared polymer solution with this way was placed into the glass vial at a certain amount and exposed to UV light (360 nm) for the different exposure times to form crosslinked hydrogels.

\subsection{Characterization of Chitosan-Based Hydrogel}

The molecular structure of chitosan-based hydrogel was verified by a Fourier transform infrared spectroscopy (FT-IR). FT-IR analysis was carried out with a Perkin Elmer Spectrum One FT-IR with attenuated total reflectance (ATR) unit and the membranes were scanned in the range of 600 and 4000 $\mathrm{cm}^{-1}$ with $4 \mathrm{~cm}^{-1}$ resolution averaging 120 scans.

\subsection{Thermal Behavior of Chitosan-Based Hydrogel}

The thermal stability of the samples chitosan-based hydrogel was determined by thermogravimetric analysis (TGA) and differential thermal analysis (DTA). To obtain weight loss profile and DTA curves of the chitosan-based hydrogel, thermal analyses were carried out performing Seiko EXSTAR 6000TGA/DTA 6300 model instrument with a heating rate of $10{ }^{\circ} \mathrm{C} \mathrm{min}^{-1}$ under a nitrogen atmosphere.

\subsection{Water Uptake Capacity}

To determine the effect of the UV time on the water uptake capacity of chitosan-based hydrogel, swelling test was carried out gravimetrically, as follows. The weighed dry hydrogels produced by using different UV exposure time immersed in distilled water at $37^{\circ} \mathrm{C}$ and stirred at $130 \mathrm{rpm}$ using orbital shaker. The swelled samples were taken from the distilled water after 24 hours and removed the adsorbed water on the surface of hydrogel by tissue paper. Then, swelled samples were weighed. The swelling ratio (SR), in percentage, was calculated by Eq. 1,

$$
\mathrm{SR}=\frac{\mathrm{W}_{\mathrm{S}}-\mathrm{W}_{\mathrm{d}}}{\mathrm{W}_{\mathrm{d}}} \times 100(\%)
$$

Where $\mathrm{W}_{\mathrm{S}}$ and $\mathrm{W}_{\mathrm{d}}$ are the weights of the swollen and dry samples, respectively.

\subsection{Cytotoxicity Assay for Chitosan-Based Hydrogel}

The chitosan-based hydrogel was sterilized by washing several times with ethanol and penicilinstreptomycin prior to the cytotoxicity tests. Cytotoxicity of hydrogel was determined using L929 cell line (Murine Fibroblasts) and WST-1 cell proliferation and viability assay. Firstly, L929 cell line at approximately $70 \%$ confluence from exponential-phase culture were washed with PBS and detached with $0.1 \%$ trypsin-EDTA. Following this, L929 fibroblasts in DMEM with a concentration of $5 \times 10^{5}$ cells $/ \mathrm{mL}$ was seeded onto the sterilized hydrogel placed into 24 -well cell culture plates and incubated for $24 \mathrm{~h}$ at $37^{\circ} \mathrm{C}$ under $5 \% \mathrm{CO}_{2}$ environment in a humidified incubator. L929 cells were seeded at the same concentration $\left(5 \times 10^{5}\right.$ cells $\left./ \mathrm{mL}\right)$ onto sample free well and used as a positive control sample. After incubation for 24 hours, $1000 \mu \mathrm{l}$ of WST-1 solution was added to each well. The mixture was further incubated for another 2 hours. Then absorbance value of WST1 for each sample was measured spectrophotometrically using a Promega GloMax ${ }^{\circledR}-$ Multi+ detection system at $450 \mathrm{~nm}$. 


\section{RESULTS AND DISCUSSION}

\subsection{Synthesis of Chitosan-Based Hydrogel by Photopolymerization}

The fabrication of biocompatible hydrogels with a high water uptake capacity is seen as a fairly important progress for biomedical applications. In current work, to form chitosan-based hydrogel, firstly photopolymerizable groups were created on the chitosan backbone by using grafting modification method (Figure 1a). After then modified acrylated chitosan having photopolymerizable groups subjected to photopolymerization with PEGDA to form chemically crosslinked hydrogel (Figure 1b). PEGDA was used as a crosslinker since it has also acrylate groups providing the reaction with CTS-g-GMA to fabricate crosslinked polymeric network, (CTS-g-GMA)-PEGDA.
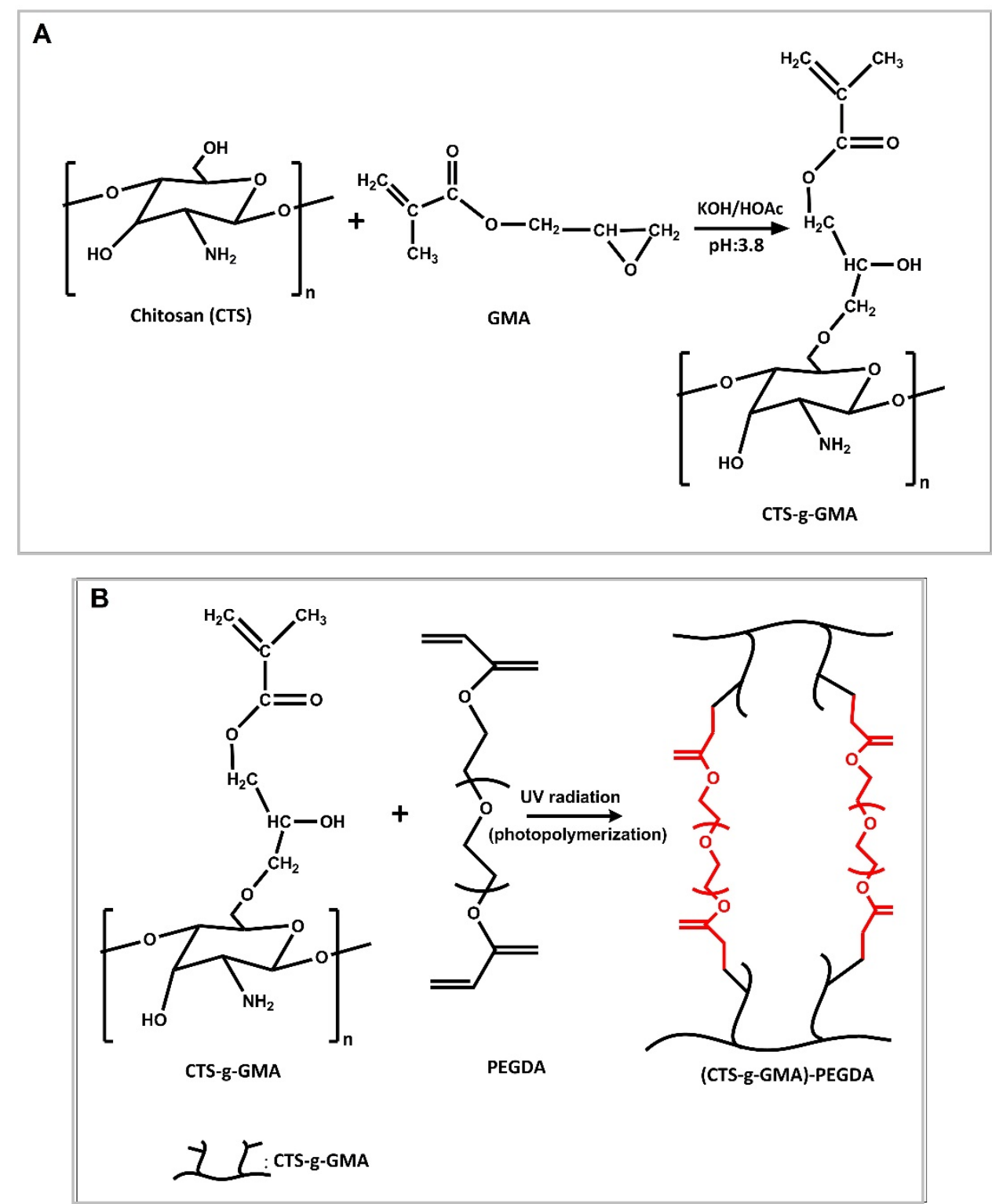

Figure 1. Schematic diagram of grafting reaction between CTS and GMA (A) and fabrication of (CTSg-GMA)-PEGDA (B) 


\subsection{Characterization of Chitosan-Based Hydrogel by Photopolymerization}

The infrared spectrum of the pure chitosan (Figure 2) shows a series of characteristic bands: (i) a broad one at $3363 \mathrm{~cm}^{-1}$ attributed to $\mathrm{OH}$ stretching frequency, which overlaps with amino stretching band in the same region; ii) typical C-H stretching vibrations at 2927 and $2873 \mathrm{~cm}^{-1}$; (iii) bands in the 1200 $800 \mathrm{~cm}^{-1}$ region corresponds to the pyranosidic ring, reflecting $\mathrm{C}-\mathrm{O}-\mathrm{C}$ and $\beta$-glycosidiclinkage as well as the $\mathrm{C}-\mathrm{O}$ related to primary and secondary alcohols [4]. The spectra of CTS-g-GMA and (CTS-gGMA)-PEGDA demonstrated some changes when compared to the pure chitosan, as observed in Figure 1 and Figure 2. For the CTS-g-GMA spectrum, the peaks at $1557 \mathrm{~cm}^{-1}$ and $1635 \mathrm{~cm}^{-1}$ correspond to $\mathrm{C}=\mathrm{C}$ stretching and $\mathrm{C}=\mathrm{O}$ vibration indicating the success of grafting of glycidyl methacrylate onto the chitosan structure $[4,19,22]$ For the (CTS-g-GMA)-PEGDA spectrum, the band at $1732 \mathrm{~cm}^{-1}$ clearly showed the presence of the carbonyl group of PEGDA owing to photopolymerization of CTS-g-GMA and PEGDA for the fabrication of chitosan- based hydrogel [23]. It is also notable that the band 1557 $\mathrm{cm}^{-1}$ corresponding to $\mathrm{C}=\mathrm{C}$ stretching disappears due to the insertion of the acrylate groups of PEGDA to CTS-g-GMA during photopolymerization process in (CTS-g-GMA)-PEGDA. Additionally, as shown in IR spectrum of the (CTS-g-GMA)-PEGDA, the crucial peaks of PEGDA could be displayed, which are at $2916 \mathrm{~cm}^{-1}$ for alkyl-CH stretching, and at $1160 \mathrm{~cm}^{-1}$ for the ether (-C-O-C-) group [21,23]. Additionally, the intensive of peak at the $2994 \mathrm{~cm}^{-1}$ corresponding $\mathrm{CH}_{2}$ vibrational mode was decreased that indicates that there was reaction between $\mathrm{CH}_{2}$ groups of CTS-g-GMA and PEGDA.

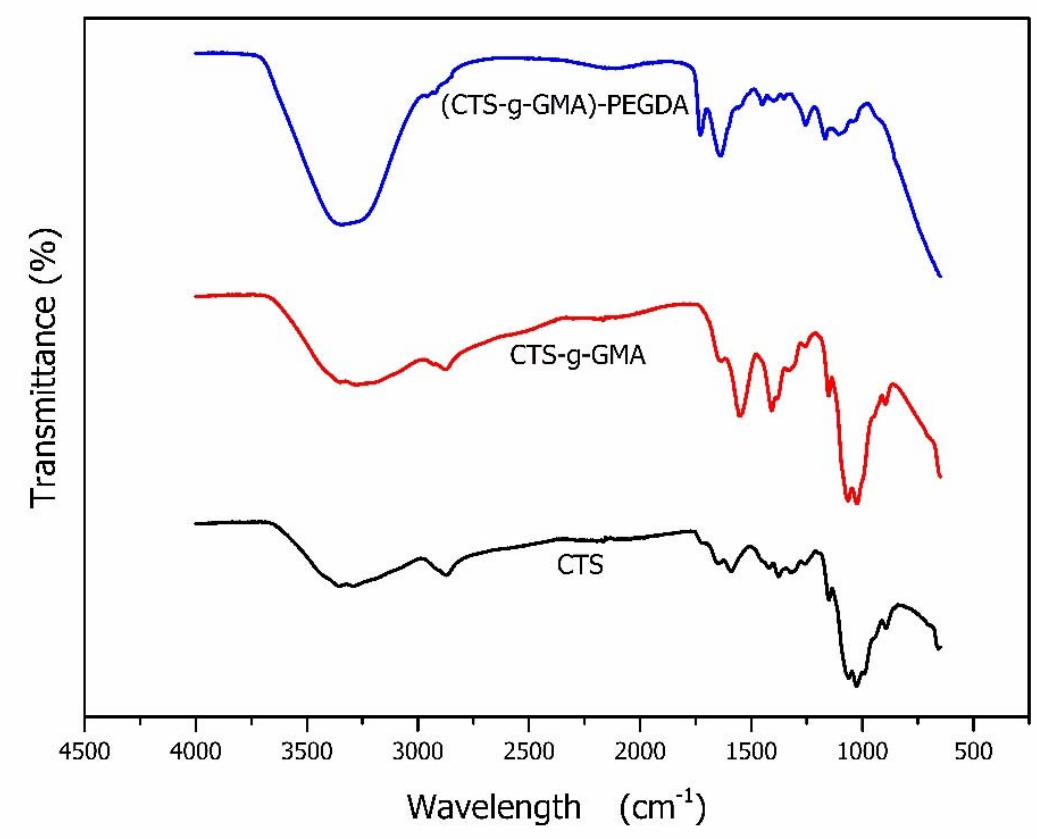

Figure 2. FT-IR spectra of chitosan, CTS-g-GMA and (CTS-g-GMA)-PEGDA hydrogels

\subsection{Thermal Performance of Photocrosslinked Chitosan-Based Hydrogel}

TGA curve for pure CTS (Figure 3a) showed two important weight losses at $68.81{ }^{\circ} \mathrm{C}$ and $308.50{ }^{\circ} \mathrm{C}$. While the first weight loss (from 26.10 to $143.25^{\circ} \mathrm{C}$ ) can be assigned to the elimination of free and bounded waters present in chitosan, the second weight-loss is due to the decomposition of cyclic moiety $[19,22,24]$. As seen from Figure 3a, CTS-g-GMA has a three well-defined weight loss region at $62.36^{\circ} \mathrm{C}, 143.14{ }^{\circ} \mathrm{C}, 275.32{ }^{\circ} \mathrm{C}$. These regions can be attributed to the water evaporation, to GMA loss, and to main degradation of grafted polymeric structure (chitosan), respectively. It can be noted that CTSg-GMA has lower thermal degradation temperature $\left(275.32^{\circ} \mathrm{C}\right)$ compared to pure $\mathrm{CTS}\left(308.50^{\circ} \mathrm{C}\right)$. 
Similarly, three weight-loss steps were also observed for (CTS-g-GMA)-PEGDA (Figure 3a). As the first step between $29.51{ }^{\circ} \mathrm{C}$ and $194.66{ }^{\circ} \mathrm{C}$ is due to entrapped water and GMA in the structure of hydrogel, the second weight-loss between $201.69^{\circ} \mathrm{C}$ and $339.82{ }^{\circ} \mathrm{C}$ corresponds to weight-loss of chitosan [25]. The last weight-loss which is appeared between $345.90{ }^{\circ} \mathrm{C}$ and $553.23{ }^{\circ} \mathrm{C}$ results from the main decomposition of the crosslinked hydrogel.
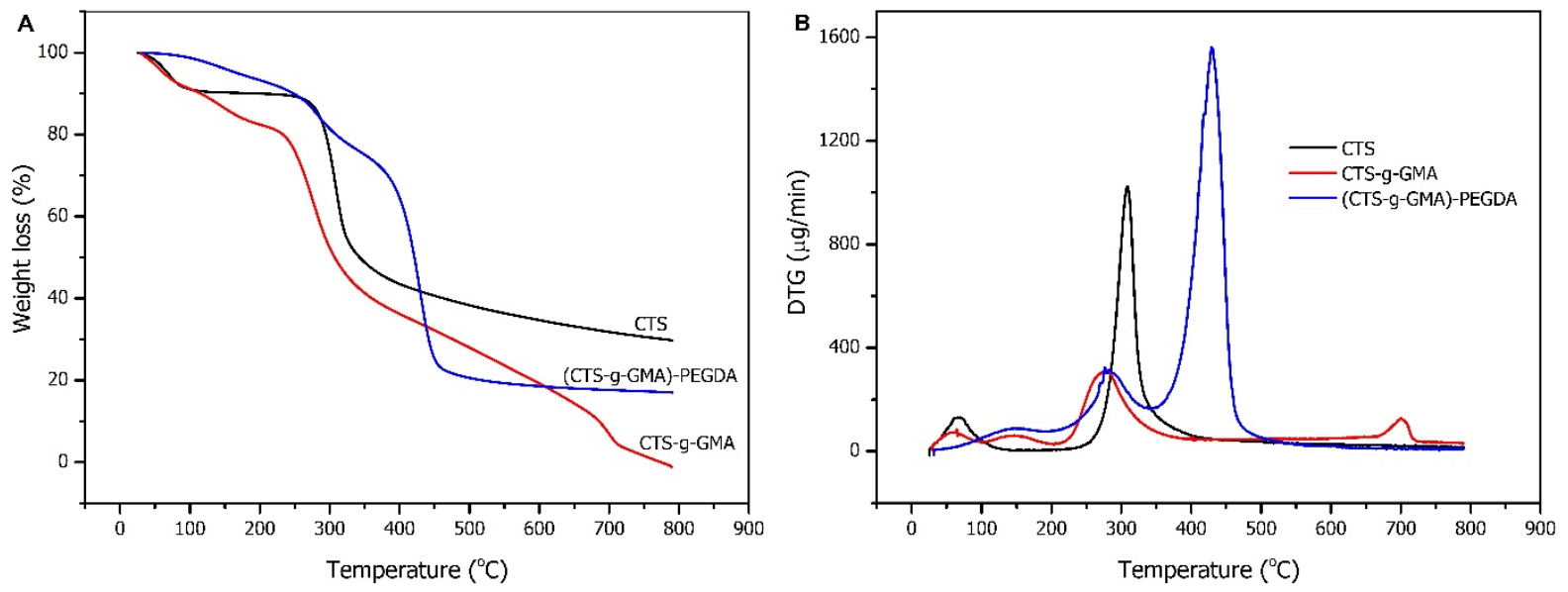

Figure 3. TGA (A) and DTG (B) thermograms of the chitosan, CTS-g-GMA and CTS-g-GMA-PEGDA hydrogel

From the results, it can be stated that thermal decomposition temperature of (CTS-g-GMA)-PEGDA $\left(429.15{ }^{\circ} \mathrm{C}\right)$ is much higher than that of CTS-g-GMA $\left(144{ }^{\circ} \mathrm{C}\right)$ and chitosan $\left(308.50{ }^{\circ} \mathrm{C}\right)$ since the structure of (CTS-g-GMA)-PEGDA is chemically cross-linked network, which provides thermal stability to hydrogel. All the TGA results are evidence that (CTS-g-GMA)-PEGDA successfully could be synthesized by using photopolymerization technique because TGA results showed the presence of all tree components in hydrogel network. Each weight-loss for CTS, CTS-g-GMA, (CTS-g-GMA)PEGDA can be also observed from derivative TG (DTG) curve related to these products (Figure $3 \mathrm{~b}$ ).

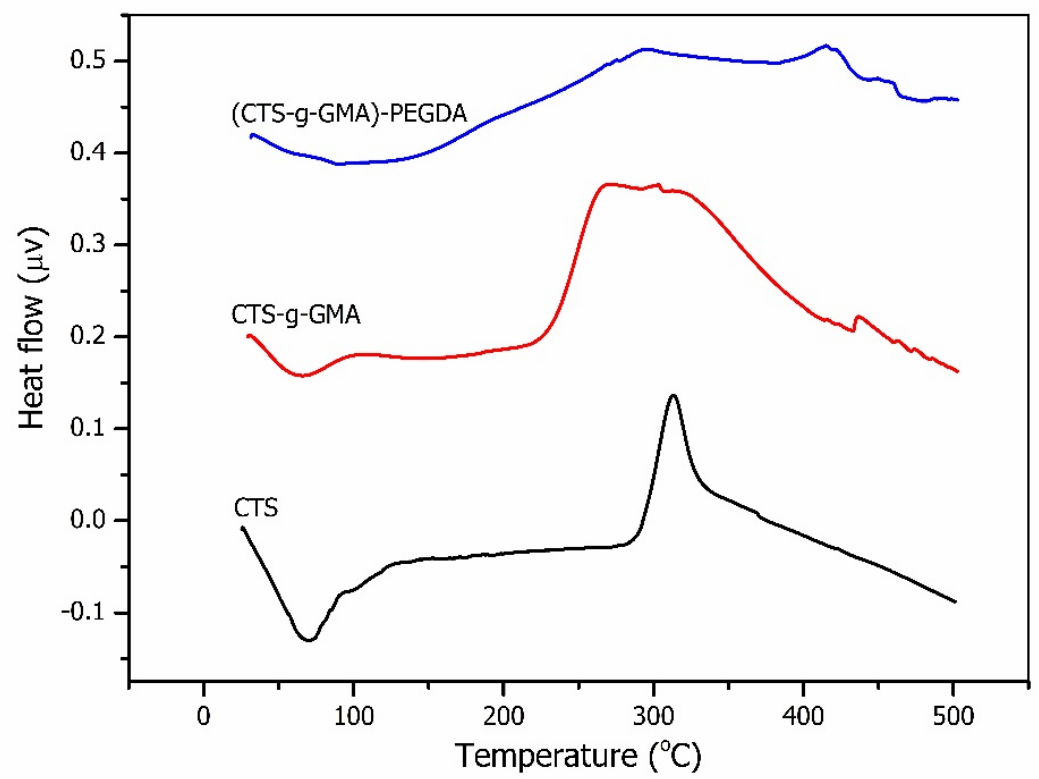

Figure 4. DTA curves of the Chitosan, CTS-g-GMA and (CTS-g-GMA)-PEGDA hydrogel 
DTA thermograms of chitosan, CTS-g-GMA and chitosan-based hydrogel were shown in Figure 4. Compared to chitosan, the introduction of acrylic groups onto chitosan backbone decreased thermal stability and degree of order. This decrease (from 308.50 to $275.32{ }^{\circ} \mathrm{C}$ ) in thermal stability of the CTSg-GMA results from the increasing chain mobility of the modified structure. In this point, it can be noted that the thermal stability could be decreased by introduction of substituent of glycidyl methacrylate groups, which could disrupt the crystalline structure of chitosan, especially through the loss of the hydrogen bonding [11]. Based upon DTA analysis, it can be stated that (CTS-g-GMA)-PEGDA has higher thermal stability than chitosan and CTS-g-GMA owing to its crosslinked structure. The occurrence of a single, broad peak in the (CTS-g-GMA)-PEGDA is indicative of attractive molecular interactions and a high degree of compatibility of both materials. DTA and TGA results confirmed each other.

\subsection{Water Uptake Capacity of Photocrosslinked Chitosan-Based Hydrogel}

To determine the effect of UV exposure time on the water uptake capacity of hydrogel, swelling tests were carried out into distilled water at $37^{\circ} \mathrm{C}$ and $130 \mathrm{rpm}$ for three different $\mathrm{UV}$ exposure times and the results were shown as a graph (Figure 5). As depicted in Figure 5, the swelling ratio of chitosan-based hydrogels decreased with increasing UV exposure time. The water uptake capacity (swelling ratio) of (CTS-g-GMA)-PEGDA obtained by UV exposure time of 8 minutes reached $\sim 622.3 \%$, which is higher than that of hydrogel obtained by UV exposure time of 20 minutes $(\sim 567.1 \%)$. These results showed that the crosslinking density has an important effect on the swelling properties of hydrogel [26,27]. The results make sense because swelling ratio depends on the crosslinking density of biomaterials. It should be noted that UV exposure time has highly important effect on the crosslinking density of polymeric network. The obtained data were given as mean $\pm \mathrm{SD}$ (standard deviation).

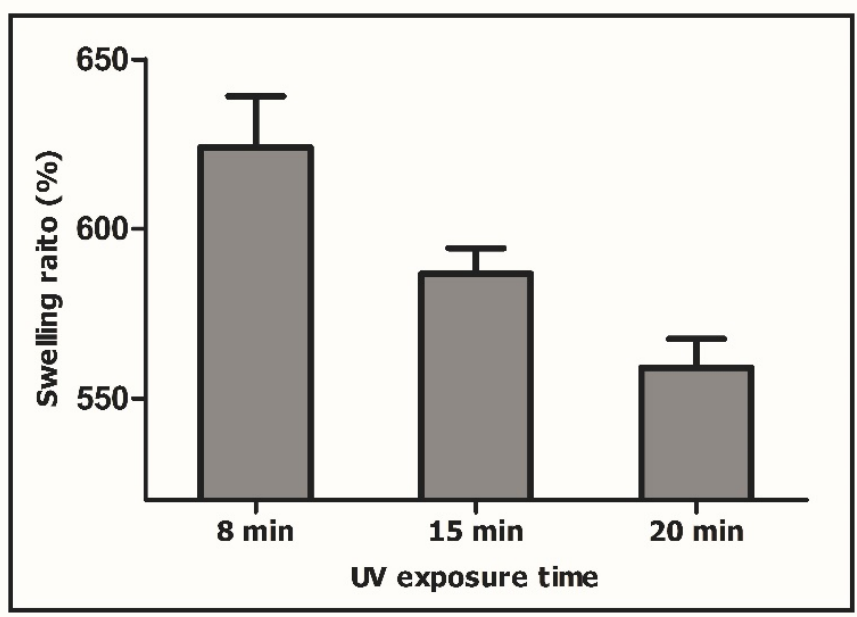

Figure 5. Swelling ratios of chitosan based hydrogels, (CTS-g-GMA)-PEGDA, obtained by different UV exposure time in distilled water at $37^{\circ} \mathrm{C}$

\subsection{Cytotoxicity of Chitosan-Based Hydrogel (Cell Proliferation and Viability Assays)}

In this study, WST-1 assay was performed to investigate the cytotoxicity of chitosan-based hydrogel. The results were presented in Figure 6. As seen in graph, it can be stated that chitosan-based hydrogel has no any cytotoxic effect for proliferation of L929 fibroblasts since there is no any significant difference between chitosan-based hydrogel and polystyrene well (control sample) in terms of the viability of L929 cells. All data were reported as mean $\pm \mathrm{SD}$ (standard deviation). 


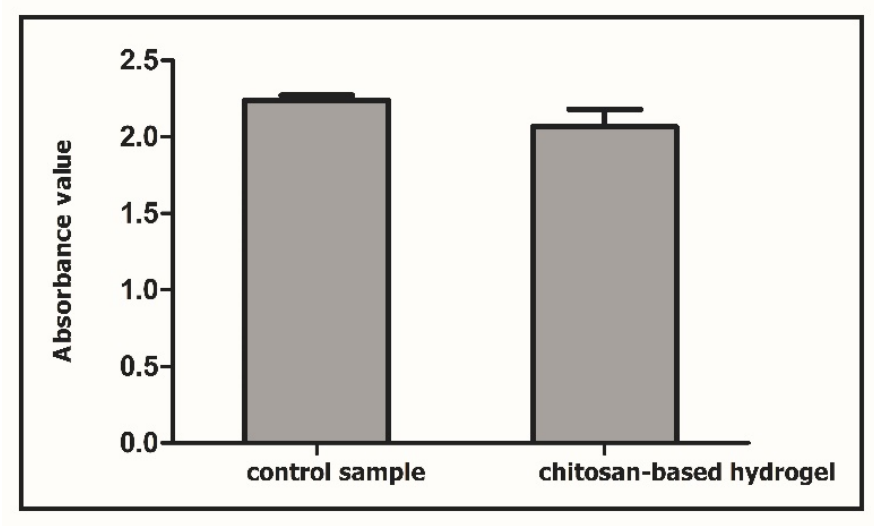

Figure 6. The viability of L929 cells on the chitosan-based hydrogel and polystyrene (control sample)

\section{CONCLUSION}

The useful and simple route for fabricating chemically crosslinked hydrogel with highly water uptake capacity and thermal stability was provided in present study. Here, the successful synthesis of a hydrogel (CTS-g-GMA)-PEGDA was carried out through a two-step reaction process: GMA was initially grafted onto the chitosan backbone (CTS-g-GMA), then PEGDA was inserted to promote photopolymerization with CTS-g-GMA to fabricate the final product (CTS-g -GMA)-PEGDA. Swelling test results showed that the increase in UV exposure time lead to increase the crosslinking density of chitosan-based hydrogels resulting in the decrease in the water uptake capacity. Biocompatibility with L929 fibroblasts of the obtained hydrogel in current study was confirmed by cytotoxicity assay. It is visualized that this chitosan-based hydrogel can be a potential material for biomedical applications involving a high water uptake capacity and thermal stability.

\section{ACKNOWLEDGEMENT}

The author acknowledges funding from the Turkish Scientific and Technological Research Council of Turkey (Project no: TUBITAK 3001- 115M624) and Marmara University, Scientific Research Project Office (FEN-A-100615-0268).

\section{REFERENCES}

[1] Hennink WE, Nostrum CF. Novel crosslinking methods to design hydrogels. Adv. Drug Del Rev., 54:13-36, 2002.

[2] Schacht EH, Polymer chemistry and hydrogel systems. J Phys: Conf Ser., 3:22, 2004.

[3] Hoare TR, Kohane DS. Hydrogels in drug delivery: Progress and challenges. Polymer, 49:19932007, 2008.

[4] Khan A, Badshah S, Airoldi C. Biosorption of some toxic metal ions by chitosan modified with glycidylmethacrylate and diethylenetriamine. Chemical Engineering Journal, 171:159-166, 2011.

[5] Pasanphan W, Rattanawongwiboon T, Rimdusit P, Piroonpan T. Radiation-induced graft copolymerization of poly(ethyleneglycol) monomethacrylate onto deoxycholate-chitosan nano particles as a drug carrier. Radiation Physics and Chemistry, 94: 199-204, 2014. 
[6] Altinisik A, Yurdakoc K. Chitosan/poly(vinyl alcohol) hydrogels for amoxicillin release. Polym. Bull., 71:759-774, 2014.

[7] Anirudhan TS, Parvathy J. Design of biopolymeric architecture with thiolated chitosan-poly (lactic acid) blend/methionine modified clay for the controlled release of amoxicillin. Polymer-Plastics Technology and Engineering, 53: 1339-1343, 2014.

[8] Lin YH, Chang CH, Wu YS, Hsu YM, Chiou SF, Chen YJ. Development of pH-responsive chitosan/heparin nanoparticles for stomach-specific anti-Helicobacter pyloritherapy. Biomaterials, 30:3332-3342, 2009.

[9] Lin YH, Tsai SC, Lai CH, Lee CH, He ZS, Tseng GC, Genipin-cross-linked fucoseechitosan/heparin nanoparticles for the eradication of Helicobacter pylori Biomaterials, 34:4466-4479, 2013.

[10] Pasanphana W, Rattanawongwiboon T, Rimdusit P, Piroonpan T. Radiation-induced graft copolymerization of poly(ethylene glycol) monomethacrylate onto deoxycholate-chitosan nanoparticles as a drug carrier. Radiation Physics and Chemistry, 94: 199-204, 2014.

[11] Maa G, Yang D, Zhou Y, Xiao M, Kennedy JF, Nie J, Preparation and characterization of watersoluble N-alkylated chitosan. Carbohydrate Polymers, 74: 121-126, 2008.

[12] Choi JS, Yoo HS. Pluronic/chitosan hydrogels containing epidermal growth factor with woundadhesive and photo-crosslinkable properties. Journal Biomed Material Research A, 95(2):564-573, 2010.

[13] Nguyen KT, West JL. Photopolymerizable hydrogels for tissue engineering applications.

Biomaterials, 23:4307-4314, 2002.

[14] Hu J, Hou Y, Park H, Choi B, Hou S, Chung A, Lee M. Visible light crosslinkable chitosan hydrogels for tissue engineering. Acta Biomaterialia, 8:1730-1738, 2012.

[15] Fertier L, Koleilat H, Stemmelen M, Giani O, Joly-Duhamel C, Lapinte V, Robin JJ. The use of renewable feedstock in UV-curable materials - A new age for polymers and green chemistry. Progress in Polymer Science, 38:932- 962, 2013.

[16] Qi Z, Xu J, Wang Z, Nie J, Ma G. Preparation and properties of photo-crosslinkable hydrogel based on photopolymerizable chitosan derivative. International Journal of Biological Macromolecules, 53:144-149, 2013.

[17] Amsden BG, Sukarto A, Knight DK, Shapka SN. Methacrylated glycol chitosan as a photopolymerizable biomaterial. Biomacromolecules, 8:3758-3766, 2007.

[18] Matsuda T, Magoshi T. Preparation of vinylated polysaccharides and photofabrication of tubular scaffolds as potential use in tissue engineering. Biomacromolecules, 3:942-950, 2002.

[19] Flores-Ramírez N, Elizalde-Peña EA, Vásquez-García SR, González-Hernández J, MartinezRuvalcaba A, Sanchez IC, Luna-Bárcenas G, Gupta RB. Characterization and degradation of functionalized chitosan with glycidyl methacrylate. J. Biomater. Sci. Polymer Edn., 16(4):473-488, 2005. 
[20] Fertier L, Koleilat H, Stemmelen M, Giani O, Joly-Duhamel C, Lapinte V, Robin JJ. The use of renewable feedstock in UV-curable materials - A new age for polymers and green chemistry. Progress in Polymer Science, 38:932-962, 2013.

[21] Zhong C, Wu J, Reinhart-King CA, Chu CC. Synthesis, characterization and cytotoxicity of photocrosslinked maleic chitosan-polyethylene glycol diacrylate hybrid hydrogels. Acta Biomaterialia, 6:3908-3918, 2010.

[22] Elizalde-Pena EA, Flores-Ramirez N, Luna-Barcenas G, Va'squez-Garcia SR, Arambula-Villa G, Garcia-Gaitan B, Rutiaga-Quinones JG, Gonzalez-Hernandez J. Synthesis and characterization of chitosan-g-glycidyl methacrylate with methyl methacrylate. European Polymer Journal, 43:3963-3969, 2007.

[23] Hwang CW, Kwak NS, Hwang TS. Preparation of poly(GMA-co-PEGDA) microbeads modified with iminodiacetic acid and their indium adsorption properties. International Journal of Biological Macromolecules, 45:499-503, 2009.

[24] Sennaroglu Bostan M, Senol M, Cig T, Peker I, Goren AC, Ozturk T, Eroglu MS. Controlled release of 5- aminosalicylicacid from chitosan based $\mathrm{pH}$ and temperature sensitive hydrogels. International Journal of Biological Macromolecules. 52:177- 183, 2013.

[25] Maa G, Zhang X, Han J, Song G, Nie J. Photo-polymeriable chitosan derivative prepared by Michael reaction of chitosan and polyethylene glycol diacrylate (PEGDA). International Journal of Biological Macromolecules, 45:499-503, 2009.

[26] Gao C, Liu M, Chen J, Zhang X. Preparation and controlled degradation of oxidized sodium alginate hydrogel. Polymer Degradation and Stability, 94:1405-1410, 2009.

[27] Hwang CM, Sant S, Masaeli M, Kachouie NN, Zamanian B, Lee SH, Khademhosseini A, Fabrication of three-dimensional porous cell-laden hydrogel for tissue engineering. Biofabrication, 2:1$12,2010$. 